\title{
An Appraisal of the Incidence of Happenstance as Leverages of Creative Instincts by Indigenous Yoruba Carvers of Western Nigeria
}

\author{
Yusuf Ayinde Abdulrasaq \\ Department of Fine and Applied Arts, Federal College of Education, Zaria, Nigeria
}

\begin{abstract}
In making sculpture composition by carving, the myriad of thoughts and actions from concept formulation to formation encapsulate an assortment of the use of techniques and elements of design, these are either in accordance to the guiding rules of traditional conventions or that of the principles of design. Aside from the import of conventions, elements of design and principles, there appears to be a fourth angle that is either often played down or overlooked entirely in the course of wood carving which cannot be ignored. This paper therefore sought to idealise the attributes and efficacy of incidence of chance as fundamental concepts to adopt in the course of actualising compositions in sculpture making by the subtractive method. The paper elucidated with some examples how, saliently, the concept of happenstance, as unplanned concepts, has contributed to the final compositions of some renowned sculpture compositions and edifices. The paper concluded by postulating that happenstance should be adopted as additional model to principles of sculpture composition and recommended to sculptors or carvers to reinforce their creative drives by being cognisance of the benefits of acceptable haphazardness as a principle of creativity to observe in sculpture composition by the carving method.
\end{abstract}

Key words: Carving, Composition, Conventions, Happenchance, Incidence.

\section{INTRODUCTION}

$\mathrm{T}$ he creation of any traditional or contemporary wood carving composition in Africa is based on the dictates of traditions or the import of academic standard that is reliant on the use of elements of design in accordance with the guiding rules of the principles of design. The trio of the concepts of traditional convention, elements and principles of design are the basis or the building blocks of artistic compositions, while Ragans (1988) asserts that "visual images are organized by rules" and that such adoption of the use of compositional guiding principles is like speaking a language using organized words.

Understanding composition making in art could be viewed from the perspective of its constituents in terms of elements and principles or by the communal dictate of the traditional conventions. These conventions may be as varied as the society in Africa, such is the peculiarity with the Yoruba traditional carvers of Western Nigeria with their unique approach often unknown to modern system of sculpture composition, with such traditional conventions, Adepegba
(2007) attests to the fact of traditional principles of Yoruba indigenous wood carving as against academic method of modern European art forms. In support of this disparity, Eyo (2008) espouses the indigenous conventions of carving by the Yoruba people of Western Nigeria in their unique representational techniques of idealising some important features of their figures. For Willet (2002) the communal principle for creating such pieces of sculptures are "culturally determined", this visual interpretation with the use of different compositional arrangement are inevitably the constituents of an artwork that both traditional African carvers and academically trained artist cannot ignore. To be unwaveringly creative therefore, an artist is disposed to rely on the creative use of exploratory drive while employing whatever technique and medium or media in a variety of ways.

With the concept of the use of the building elements and the guiding principles of design as well culturally sanctioned models, there is the notion that the cravings for the creation of compositions place it on artists who continue to seek an aura of creative deluge of uniquely crafted sculptures. Whether of abstract collections or vivid realistic compositions, where the artists seek to mediate between the widely objectively known or obscured in meanings, a creative outlay comes in layers of stages from the incubation, schematic, exploratory and actualisation to presentation. Along this linear basis of gradient of concept of production, whatever comes from the artist becomes a little bit of planned assurances and a little bit of whatever results from a creative encounter (Cambridge Dictionary 2017).

The postulation that the totality of the outcome of a design is all humanly induced is to be oblivion of what nature has as inducements in complementing human efforts. it is known that no matter how we strive to come up with an artistic composition or design, there are some intervening phenomena that will always alter the final outcome of a design however little or micro. Those factors suppressing or aggravating the human factor in the actualization of a design are quite in number. From the characteristics of the structure of the material employed to the limitations of the artist's finger dexterity in the way tools are manipulated as well as the undeniable acceptance of the tools markings involved, the context of the final outlay of the work produced are all just the 
normal phenomena that may simply alter the course of the original thoughts of a design beyond humans (Jari and Nwankwo, 2000).

For a creative encounter that stems from the basis of acceptance of external influence outside the parameters of a preplanned design, the significance of the unique acceptance calls for more than just a cursory look. To substantiate this assertion, Jari and Nwankwo (2000) affirm that as Man attempts to order the world around him through design, forces intervene to aid, disrupt or sway his course of creative exploration, and in the end, what gets realized is hardly the precise thing the mind conceived as man is never in total control. Like in a litmus test of how the force of acid changes the substance's colour to red, planned action is, usually, set on collision with normal and unplanned realities in an analogy of the impact of the usual and accidental occurrences. The concept of chance that is commonly ignored, therefore, can be adopted as an acceptance of the expected and the unexpected when juxtaposed with attending surprises. This paper therefore espouses the concept of unexpected, which has happened, as leverage to creative exploration in wood carving. So this paper is focused on the relative benefits for adopting the instances of incidence with its unplanned consequences and happenstance as desirable elements of carving composition.

Concept of Commonly Ignored Realities in Happenstance as basis of carving

The concept of the virtue of happenstance bear a hallmark of a series of the unexpected often encountered during exploration. The fact of its unpredictability largely points towards many of the unplanned occurrences in the course of making a composition of wood carving, on the other hand, the instance of the endless opportunities in happenstance, often beyond human control, makes the adoption of the unexpected occurrences as an unbridled phenomenon in an aura of boundless platform of creativity even despite the knowledge of its impending occurrence (Dictionary .com, 2017).

As against proactive creative reasoning in accepting the unexpected in the course of carving, the lack of capacity to handle a clearly commonly encountered event is a restrictive weakness on the ability of human. In life, some phenomena comes with some limitations that are clearly beyond what human can sway its tide however small, but the acceptance of the influences of such limitations with the attending surprises become a vista in the scheme of exploratory drives. When the concept of the unexpected is juxtaposed with the attending surprises of the happenstance, both are almost of the same status when adapted for functional benefits especially in the area of making composition.

Array of Salient Attractions of the Unexpected or Natural Incidents that has occurred in Art

Whether coming from the uncontrollable influences of the characteristics of a medium adopted in a composition or the sudden twist of fate in the instances of form alteration, the incidence of unplanned instances has become common features of, possibly, all artistic creations. From the said inherent natural structure of the materials used in a composition to the limitations of tools handling and the rest, there appears to be an unending aura of external strains sculptors or carvers need to contend with in part or in whole while striving to fashion something novel. For example, Brein (1968) asserts that there are design potentials in applying pigment in uncommon ways such that "Some are the result of bringing together materials that react with each other".

In the field of art, the architects are, perhaps one group that gives in too much to preparatory meticulous mathematical outlay of designs, and with this total conservatism about following a planned design, one should think that whatever is built should be a hundred percent exalt copy of what was drawn, but that is never the case as they, each time, have to grabble with the exigency of the site layout thereby resulting in the variations of "as drawn" and the fairly unplanned "as built". A vivid example is with the "Leaning Tower of Pissa" (Fig 1). With its unintended tilt, the structure has become a world's renowned structure by sheer virtue of a debilitating tilt that has almost caused its fall except for the artificial counter balancing with lead weight in 1998 (Yallabok.com, 2017).

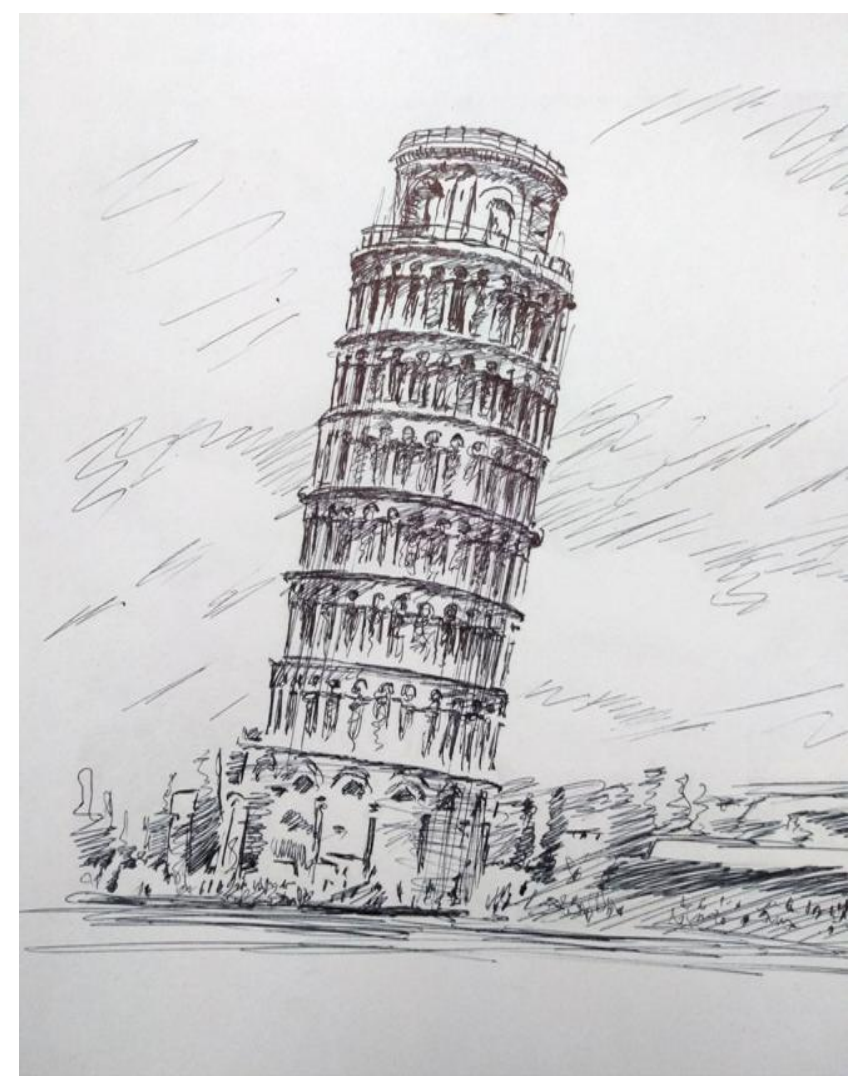

Fig 1, An illustration of the Leaning Tower of Pissa, Built around, source ; Yusuf A. A. 
In another instance of virtue turned viral, Kuper (2010) reports of the theft of "Mona Lisa" in 1911 which had caused the turn of event for the status of the painting, after the theft, queues formed outside the museum and people were streaming in to see the empty space where Mona Lisa had once hung. Prior to the theft by the burglar known as Peruggia, the painting had never caused such a stir, McKenzie (2013) while quoting Noah Charney, agrees that if a different Leonardo's works had been stolen and was able to cause such a hype, then that would have been the most famous work rather than the Mona Lisa.

For the adoption of unplanned structure of material, the thoughts of Michelangelo Buonarroti, vividly comes to mind with his marble carving composition. Michelangelo was said to be greatly influenced by the accidental structure of the boulders in Carrara by relying solely on the incidence of natural chance of the structure of the marble to dictate his composition. The veracity of the various inherent unique shapes in the quarry became a reservoir of exploration for him as his method of revealing what he perceived within the shape as naturally inherent in the rock (Michelangelo.net, 2021).

With the array of works produced by the drift-wood workers, the luck of the ocean current and the discovery of what it carries make for a myriad of choice of a direct carving approach of the inherent forms in the wood. The same goes for the trappings of twigs and stump wood sculpture compositions. The uniqueness of the acceptance on the initial shapes of the twigs is an unfettered method of how chance in natural occurrence can be made to inspire sculpture composition as seen in "Wingless" (Fig 2).

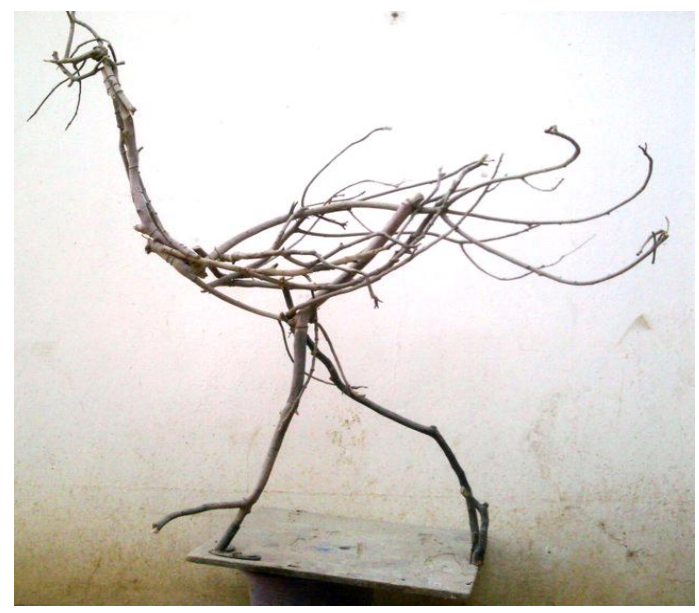

Fig 2, "Wingless", twigs, by Yusuf A.A. 2001 Image credit: Yusuf A. A.

In the same vein "Freedom" (fig. 3) emerged from the winged branches of a wind felled tree, the instance of the somewhat destructive gale is a fact that has charted the course of creativity by first bringing down the log, the second being in the creative capacity of the sculptor to have visualiased the librated form within the two-pronged branches emanating from the tree. For sculptors who crave for the excitement of direct carving, the distinctiveness of a stunted growth, broken branches, naturally split sections, cracks and incidence of termite bite become a dynamic creative impulse in the creation of forms so rarely difficult to replicate as in "Freedom" ( Fig 3).

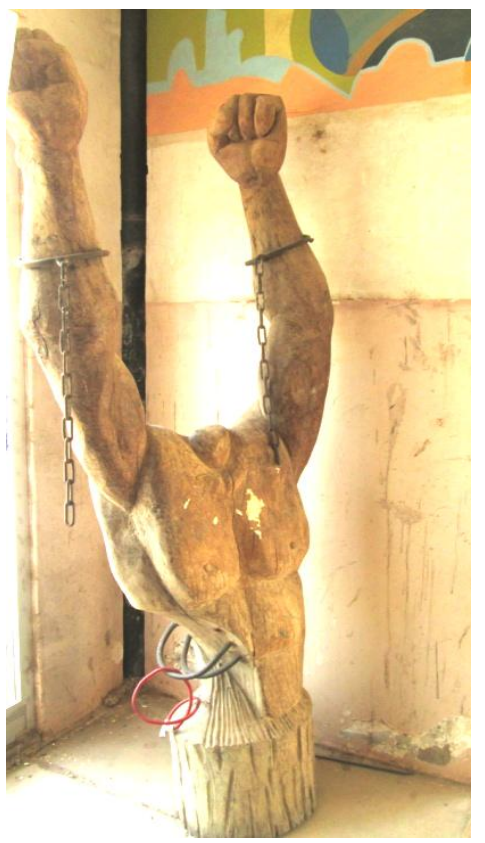

Fig 3, "Freedom" wood and metal affixtures, artist unknown, Image credit: Yusuf A. A.

For the sculptors who subtlety get inspired by the new found forms in discarded motor parts junks, the simple adoption of the former kinetic objects as basis of inspiration in assemblage is an upbeat of the acceptance of the unexpected in forming compositions, with the "Acrobat" (Fig. 4), an old broken scissors, flywheel bolts and nuts are a conjugation of formerly incongruent forms as now harmonized in one piece.

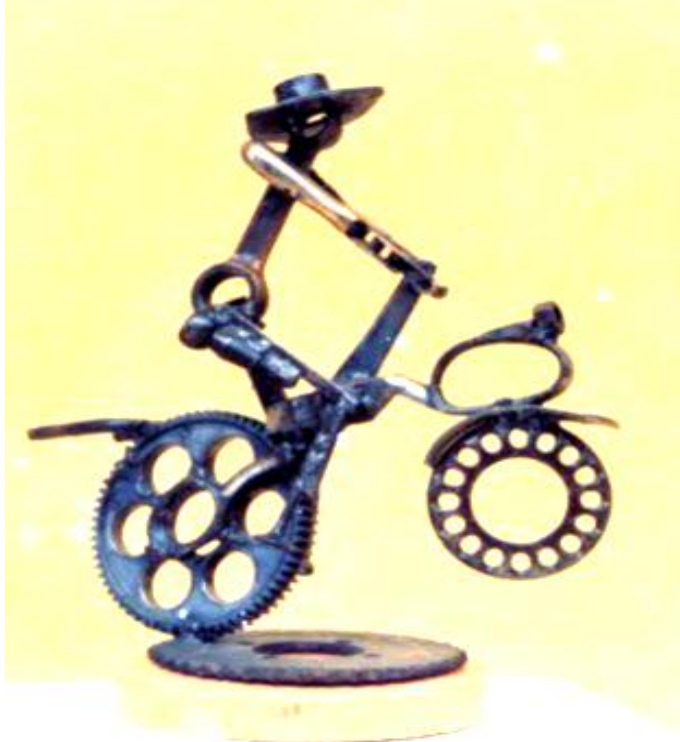

Fig 4, "The Acrobat", metal, $25 \mathrm{~cm}$ high, Yusuf A. A. Image credit: Yusuf A. A. 
The merit of the adoption of the unexpected for making composition in sculpture is not limited to the inherent material structure alone, if by chance, there is an accident of damaged or lost parts of a sculpture during the process of composition, it should not be a debilitating effect on the creative drive to produce a piece, rather, such deformations should be promptly adopted for the reordering of forms, this is visible with "Black Power"(Fig 5) a sculpture whose other adjoining figures were lost during the period of the casting in the studio but the remaining two have continued to survive since its installation in 1983. The work is still at the Faculty of Medicine, Ahmadu Bello University, Zaria, such unusual acceptance of compositional change is only in the realm of the concept of benefit of accident in the course of making design.

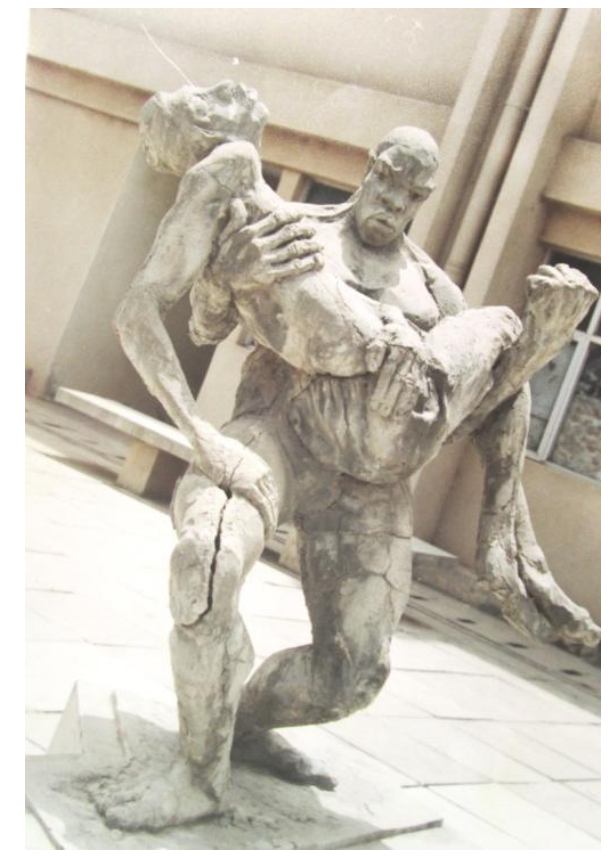

F ig 5. "Black Power" by Edward Ejembi, Concrete, Faculty of Medicine, Ahmadu Bello University Zaria. Image credit: Yusuf A. A.

Natural forms of wood as impetus for planned and unplanned composition in wood carving

In Africa, there is no doubt about the preponderance of wood as a medium of artistic expression with the technique of carving, as a traditional material, the abundance supply of the material from the major forest regions of the continent has been the incentive for this, Yusuf (2021) asserts that the medium is, perhaps, the most common carving material of all and possibly one of the oldest for traditional sculpture, this is so because the continent is blessed with an expanse of forests beaming with a steady production of the medium.

Even from the perspective of history, the comparatively few surviving numbers of carved wooden pieces from ages has not distorted the acknowledgement of the predominance of the material. The prevalence of the material despite its relatively little quantity from the past is set against the fact of its perishability by environmental impact. To idealise this
Editorial team (2019) states that a lot of traditional African artworks have not survived from time because they were made from perishable materials. As to this, despite being degraded from time by termites and other insects, moisture as well as fungi, these have not debased the fact that wood, as an indigenous medium, is of greater utility in traditional artistic renditions

Wood for carving is a product, mostly, of the stem of trees that has matured over a period of time since its growth. The choice of a particular piece to carve is often, basically, a matter of the preference of the carver based largely on a number of factors ranging from the size of work required to composition and aesthetics or functional utilities. That form follows function is a concept borne in mind preparatory for what is intended to be carved by the carver, the need for the piece will definitely require a particular approach of production.

Being basically a reductive or subtractive method where the carver chips away from a block of wood, there is the onus that the carver rather follows a direct or the indirect approach, Indirect approach meaning that there can be a preconceived or preliminary model to be followed as transitional piece to influence the intended one while the direct method is a straight forward on-the-block process where the shape emerges as the carver works on. With the two methods of approach, the direct carving method is the age-long traditional and conventional process while the indirect is more of a modern method (Yusuf. 2021).

With the two approaches highlighted above, there appears to be a third unique process that has not been given prominence. Though the process is a variant of the direct method of carving, the basis of the approach is on the natural shape of the wood as inspiration for the intended composition. While the carver is expected to work on the material directly, the inherent shape deducible from the visual imagery visible on the wood is to be further enhanced by refinement. This is simply a concept of the creative instinct of the carver to be able to tease out the emerging form in the wood as found naturally as nature-induced design, happenstance, form-ofcoincidence or accident in design. By this the inherent shape of the wood to be carved is relied on as a suggestive lead to the shape to be so derived from the wood.

Often by their natural growth or twist of fate in physical deformation, the knots in wood, wobble and pronged branches, splits and holes in trunks as well as hollowed-outs are all to be taken as basis for images to be further enhanced. The bizarreness associated with this natural probability of occurrence in wood is not only in the strange structures of the material alone, but in the luck or peradventure of the discovery in the jungle as well.

\section{MATTERS AND DISCUSSIONS}

The indigenous Yoruba carvers can be found in the South West of Nigeria, they are known for quite a number of many 
traditional art and crafts. Among these, wood carving is said to take a centre stage as a means of their engagement in traditional religion and production of object of utilities (Willet, 2008). As is the usual approach to carving, both the direct and the indirect techniques are employed in the production of their wood carvings. From many of their traditional masks to wooden statues and other utilities, the determinant factors in the creation of forms, though may be created by the direct carving approach, the functionalities of quite a number of their utilities are determinants in the choice of wood for carving. As to this, the coincidence of the natural forms of wood is germane and peculiar to the carving of some pieces. In addition, the incidence of chance of discovery is also is peculiar factor in the course of search for the appropriate shape.

Incidence of two-prong-branched wood as inspiration for indigenous wooden utilities by Yoruba carvers

The search for wood as a medium for carving is primarily hinged on the forms of the intended composition, be it a piece of casual aesthetics or practical functional utility, the mode of carving is also based on established procedures of either confronting the work by the direct or indirect approach. With the two approaches, some objects for aesthetics and functional utilities are best suited with the direct approach. this is also hinged on the probability of happenstance of procurement. Of particular refrence are all the wooden utilities that demand being of "Y" ended shape. Variously called "Paluga" or "Paluka" (Fig. 6), the "Y" shaped wooden pieces are of functional status desirable as house posts "Opo-ile", sling wood for catapults "Kannan-kannan" rafters "Eke" and shed props "Opo pepe".

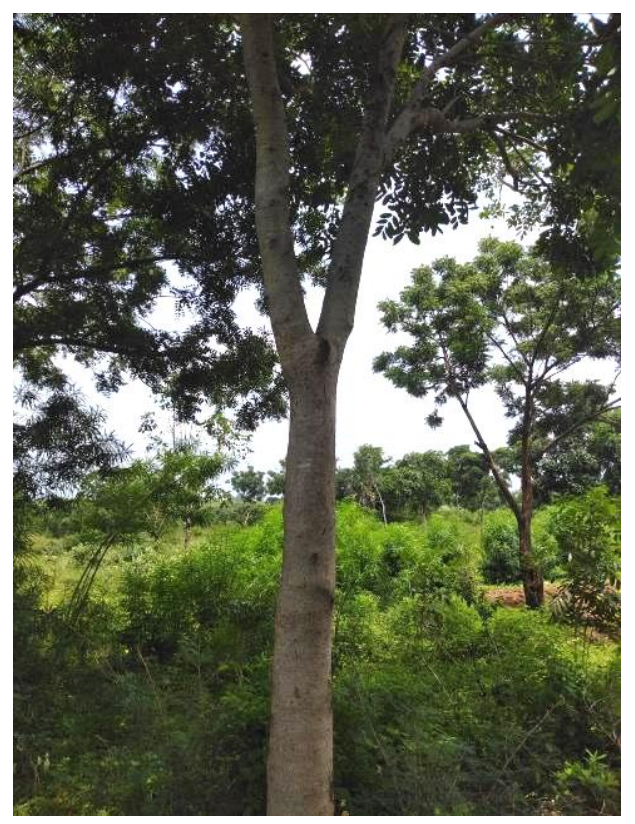

Fig. 6, A tree with two branches. Image credit: Yusuf A. A.

The instance of carving as dictated by form is a task beyond the mere rubrics of composition making in wood by indegenous Yoruba carvers, as noted earlier, some objects are said to be of some specific utilities, especially the "Y" ended carved pieces with special uses as demanded by their special branches, aside the luck of the inherent natural forms of the wood, the carver is confronted by a second probability of happenstance of the discovery of the piece in the forest, this is so because the special pieces, with their branches are hidden among other numerous pieces that make up the forest.

For the special tasks that other forms of carved wood may not serve, "eke", the rafter needs the prong to sit on the wall, the sling, "kannan-kannan" needs the "Y" shape (Fig. 7) as the target and flip space for the stone intended to hunt down a bird. For the tents, house posts or wooden pillars, "opo", the "Y" ends are direct anchorages for the crossbars. That all these "Y" shapes are indispensable for their utilities make them non replaceable.

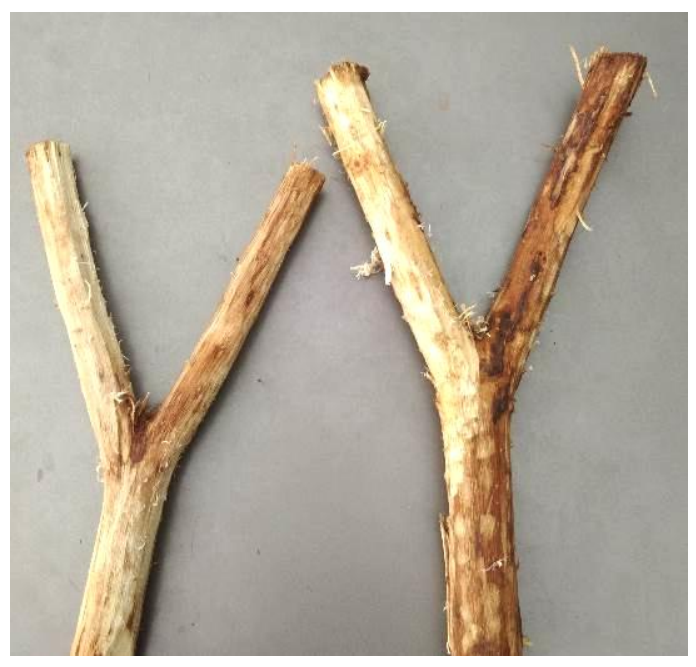

F ig 7. "Paluka or Paluga" two-pronged wood. Image credit: Yusuf A. A.

Having identified the necessity of "Y" shape contortions in wood as prerequisite to the carving of certain objects of aesthetics and functionality, the incidence of happenstance, chance, luck accident of discovery and sheer nature of growth are all parts of the embodiments of techniques and processes for carving certain wooden pieces by indegenous Yoruba carvers of Western Nigeria. the adoption of the practice of acceptance of happenstance is of great merit in helping to eliminate the psychological burden of the period of incubation and conceptualization as this is already an established notion with the cultural convention.

\section{CONCLUSION}

Whether it is the adoption of the wriggling of cloths for accidental crackles in batik, raku design in ceramics, cracks and smoke stains in pottery, natural splitting up of wood, cup shake, heart shake and wobbling growth as well as chance of discoveries of drift wood, the thrust of how incidence or chance comes to play as leverages for composition is unequivocal. Psychologists themselves attest to the efficacy of haphazardness with the upholding of "Ha ha" feelings of 
contentment when answers to a serious questions are stumbled on without plans. The accepted lost of details in the transition of clay model from mould-taking to casting is a phenomenon that modern sculptors have also come to terms with. All these are pointers to the significance of happenstance.

Since the essence of composition is for its functional and aesthetics appreciation, whatever invalidates or evacuates the barriers of exploration in whatever form becomes a welcome idea, be it the acceptance of the unthinkable, the bizarre approach, unplanned methods, the unrehearsed, the normal and abnormal that are often ignored, all these pointing to a collection of an uncanny approaches to be espoused for their exceptional benefits.

\section{RECOMMENDATIONS}

From the array of unusual adoptions and adaptations, it is now imperative for sculptors, especially carvers, and other artists alike to further sharpen their inquisitive skills as well as be on the lookout for any adaptable forms and techniques that could be promptly adopted as leverages for and in composition. The following are therefore recommended.

- Professionals in the field of art should see the issue of happenstance as a phenomenon that should be benefited from to further enhance inventiveness.

- Mechanism should be in place for purposeful trainings in the adoption of the unexpected for creative compositions.

- Sculptors and other artists alike need to take a closer look at familiar objects that have, often, been taken

\author{
for granted before to conceptualise new \\ compositions.
}

\section{REFERENCES}

[1] Adepegba, K. (2007). Contemporary Yoruba Wood Carving, Ikorodu, Aramanda Creations.

[2] Cambridge dictionary (2017). More by Accident than by Design: because of luck and not because of skills or organisation, http://dictionary.cambridge.org/dictionary/english/more-byaccident-than-design, viewed 25-03-17

[3] Dictionary .com (2017) limitation, http://www.dictionary.com/browse/limitation.

[4] Editorial team (2019). Africa's Art Material, https://thinkafrica.net/african-art-materials/

[5] Eyo, E. (2008). Master pieces of Nigerian art, Abuja, Federal Ministry of Information and Communication.

[6] Houpt, S. (2006). Museum of the missing: A History of Art Theft, New York, Sterling Pub. Co.

[7] Jari, J. \& Nwankwo A. (2000). Accident and Design: Gani Odutokun and His Influence, London, School of Oriental Studies p12-20

[8] McKenzie, S. (2013). Mona Lisa: The theft that created a legend, http://edition.cnn.com/2013/11/18/world/europe/mona-lisa-thetheft/

[9] Michelangelo.net (2021). Atlas Slave, http://www.michelangelo.net/atlas-slave/

[10] Ragans, R. (1988). Art Talk, Calofornial, Glenceo Publishing. P5

[11] Yallabok.com (2017). The Leaning Tower of Pissa, https://www.google.com.ng/search?q=leaning+tower+of+pisa\&rlz = viewed 25-03-17

[12] Yusuf A. A. (2021). Fundamentals of carving as a practice beyond the focus on wood in a book: Entrepreneurship in vocational and technical education, Ed; Okoli, T. O., Nwike M. C and Ofodile, S. N. Umunze, Cosinachiz Resource

[13] Willet, F. (2002). African Art, London, Thames and Hudson Worl. 